\title{
Professor Prithvi Mruthyunjaya: working together to move the field forward and better help our patients
}

Submitted Sep 17, 2019. Accepted for publication Sep 26, 2019.

doi: 10.21037/atm.2019.09.148

View this article at: http://dx.doi.org/10.21037/atm.2019.09.148

\section{Editor's note}

Between August $17^{\text {th }}$ and $18^{\text {th }}$, the 1 st CIMS International Medical Summit 2019 was successfully held in Beijing, China, with a focus on the hot topics in obstetrics, lung cancer, gastrointestinal cancer, proton beam therapy, neuro-oncology, neurosurgery and other areas. During the conference, we had the pleasure of interviewing Prof. Prithvi Mruthyunjaya (Figure 1), the associate professor of Ophthalmology from Stanford University, who shared his perspective on the ocular impact of systemic cancers.

\section{Expert's introduction}

Prithvi Mruthyunjaya (Figure 2), MD, MHS, is the Associate Professor of Ophthalmology at Stanford University, a member of the Vitreoretinal Surgery Service, and is the new Director of Ocular Oncology at the Byers Eye Institute. He is a board-certified ophthalmologist who has completed two prestigious fellowships: the first in Vitreoretinal Surgery at Duke University and the second in Ocular Oncology at Moorfields Eye Hospital in London, England.

\section{Interview (Figure 3)}

ATM: You had an excellent speech on "Ocular impact from systemic cancers" at the conference. Could you briefly introduce your main points on this topic to our readers?

Prof. Mruthyunjaya: I am an ophthalmologist and an ocular oncologist; I specialize in the care of patients that have systemic cancers that then spread to the eye. My talk today discusses the very frequent incidents of metastases from systemic cancers, as they present in the eye to ophthalmologists. Patients can present with vision loss and vision changes. We want to make the audience aware of some of these findings as well as new therapeutic options for patients with cancers including targeted agents and monoclonal antibodies. I want to make clear that

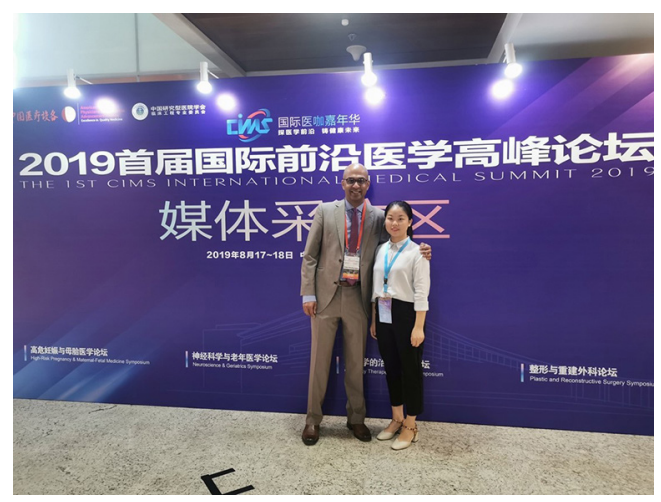

Figure 1 Picture of Prof. Mruthyunjaya and an AME science editor.

some of the toxicities unique to these medications can be vision-threatening and even blinding, which makes it very challenging for patients who are dealing with systemic cancers to also have to deal with the ocular side effects. This is the purpose of the talk.

ATM: Would you comment on the current development of clinical management guided by molecular insight?

Prof. Mruthyunjaya: One of the diseases that I specialize in is the treatment of ocular melanoma. This is a rather rare disease that differs from cutaneous or skin melanoma. We reviewed the data from China and found that not only is the incidence of this disease low in China, but survival is actually much higher in affected Chinese patients than that in the western countries. We will talk about the role of doing biopsies of these tumors and obtaining genetic information that now can allow us to segregate the risk of patients developing metastatic disease. So now, we can better focus our attention on the patients with higher risk diseases and see how we can help to make their lives better with more effective treatment. 


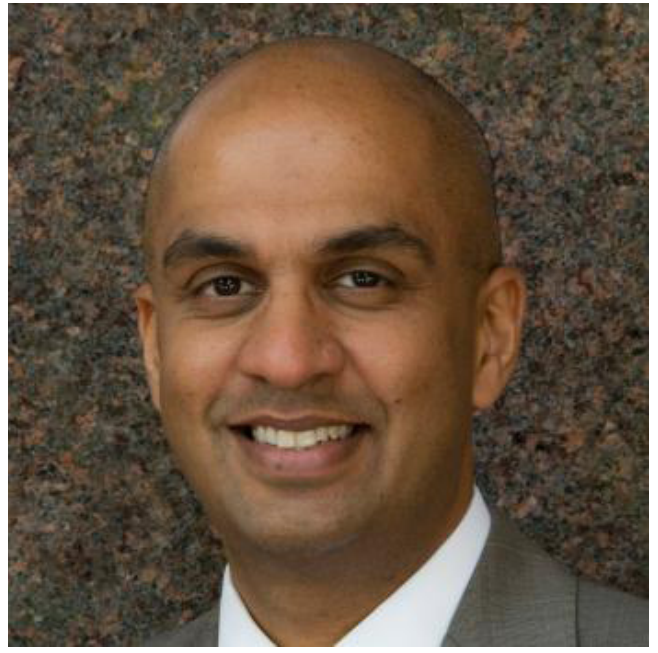

Figure 2 Prithvi Mruthyunjaya MD, MHS.

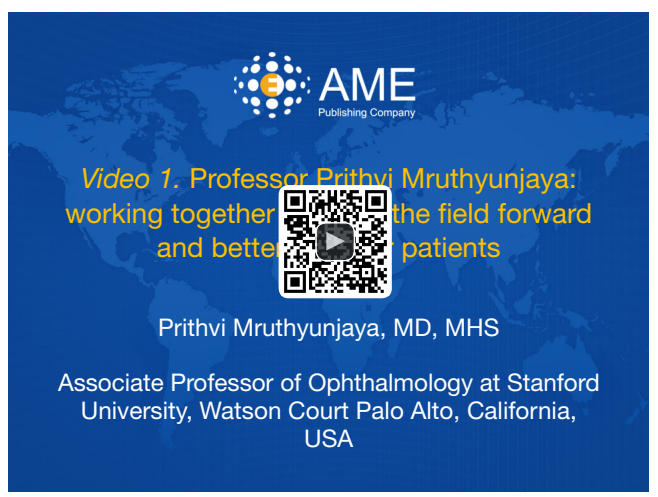

Figure 3 Professor Prithvi Mruthyunjaya: working together to move the field forward and better help our patients (1). Available online: http://www.asvide.com/watch/33015

\section{ATM: Have you come across any obstacles in your career or research? What has been the driving force for you to overcome these challenges and move forward?}

Prof. Mruthyunjaya: I think we can all say that there are challenges in moving science forward. I think the most important thing for me is building connections and networks across the United States and the world. And I think some of the challenge is to have other doctors who treat very rare diseases like I do come together and share their information and experience going forward. But the hardest thing is to get people to work together; the best part of what happens is that when they do work together, then we can actually move the field forward and better help our patients.

\section{ATM: Could you please share with us one of the most impressive or challenging cases in your clinical practice?}

Prof. Mruthyunjaya: The real challenges I think come from patients that have systemic cancers and then develop side effects from the treatment affecting their eyes. The eyes are so important from a quality of life standpoint. When you're sick from a disease, but then can't see your children or your family or do your work because of that disease, it affects your wellbeing and your quality of life even worse. I had a patient the other day who is in his 80's and was being treated for metastatic melanoma that then spread into his eyes, severely blocking his blood vessels and leaving him almost blind. So now we have to adjust the work with oncologist medications and try to unblock the vessels in his eyes, and I think we can do it. I think the challenge is how do we identify these at-risk patients and prevent these conditions rather than having to treat them.

\section{ATM: Do you like any outdoor sports outside of work?}

Prof. Mruthyunjaya: Yeah. I enjoy bicycling and spending time outside with my children. They keep me pretty active.

\section{Acknowledgments}

We would like to express our sincerest gratitude to Prof. Prithvi Mruthyunjaya for sharing his insights and opinions with us.

\section{Footnote}

Conflicts of Interest: The authors have no conflicts of interest to declare.

\section{References}

1. Qiu A, Zhou A. Professor Prithvi Mruthyunjaya: working together to move the field forward and better help our patients. Asvide 2019;6:330. Available online: http://www. asvide.com/watch/33015

(Science Editors: Abby Qiu, Angelia Zhou, ATM, editor@atmjournal.org)

(Language Editor: John Ayric Gray, AME Publishing Company)

Cite this article as: Qiu A, Zhou A. Professor Prithvi Mruthyunjaya: working together to move the field forward and better help our patients. Ann Transl Med 2019;7(22):709. doi: 10.21037/atm.2019.09.148 